\title{
Detection of localized damage by eddy currents technique
}

\author{
A. Aoukili, A. Khamlichi \\ Communications Systems and Detection Laboratory \\ Abdelmalek Essaadi University, Tetouan 93002, Morocco
}

\begin{abstract}
Non destructive evaluation techniques based on eddy currents (EC) are largely used for quality control of the castings in a lot of industries. The principle of detection by EC consists in using an adequate inductive coil to generate them by a variable magnetic field, and measuring their effects by using one or several sensors. These effects result from the interaction between the induced magnetic field and the excited conductive material. A local variation of the physical properties or geometry of the tested sample, due to a singularity or a flaw, causes a modification of the EC distribution, enabling thus detection. In order to optimize the capacity of defect revealing by means of EC based probes, an accurate modelling of the problem is essential. This can be used to perform simulation of the EC distribution under different circumstances and to analyze the EC sensitivity to the various implicated parameters. In this work, the modelling of EC is made by using the finite element method. Using a B-scan strategy was used, detection of a small defect having the shape of an open cavity is shown to be correctly indicated via monitoring variations of the induced voltage in the receiver coil.
\end{abstract}

\section{Introduction}

Non destructive evaluation (NDE) techniques based on electromagnetic methods are frequently used for damage detection in conducting metallic parts. These types of inspection methods are largely employed for quality control of for example the castings in car and oil industries, with the aim to detect surface and subsurface discontinuities in the tested parts. The electromagnetic based methods incorporate mainly two categories: magnetic particle inspection (MPI) [1] and eddy currents (EC) [2]. In the MPI based method, the process consists of putting a magnetic field into a ferromagnetic part such as iron, nickel, cobalt, and some of their alloys. The piece can like this be magnetized by direct or indirect magnetization. Direct magnetization occurs when the electric current is passed through the test object and a magnetic field is created in the material. Indirect magnetization occurs when no electric current is passed through the test object, but a magnetic field is applied from an outside source. The magnetic lines of force are perpendicular to the direction of the electric current which may take the form of either alternating current or direct current. The presence of a surface or subsurface discontinuity in the material allows the magnetic flux to leak. If ferrous iron particles are applied to a part with an area experiencing flux leakage, the particles will build up at this area and form what is called an indication. The indication can then be handled to determine what may have caused it.

The need to use ferrous particles makes the MPI less attractive than EC based methods. Moreover, EC testing uses electromagnetic induction to detect flaws in conductive materials that are not made necessary from ferromagnetic materials. The surface of the conductive material should however be accessible and the finish of the material should be enough smooth to not disturb the readings. In this technique, the depth of penetration into the material is controlled by the conductivity of the tested material and the frequency used. Flaws that lie parallel to the probe may not be detectable, so proper orientation of the probes should be arranged.

In a standard EC testing a coil carrying current is placed in proximity to the test specimen. The alternating current in the coil generates variable magnetic field which interacts with test specimen and generates EC. Variations in the phase and magnitude of these EC can be monitored using a second receiver coil or by measuring changes appearing in the current flowing in the primary excitation coil $[3,4]$. Variations in the electrical conductivity or magnetic permeability of the test object, or the presence of any flaws, will cause a change in these EC and as a consequence changes in the phase and amplitude of the measured current will be observed [5]. This constitutes the basis of standard EC inspection which is the most widely used EC technique in practice. 
The main advantage of EC testing is that they can detect very small cracks in or near the surface of the conductive material. Use of the eddy currents as an inspection technique is expected to go forward because of its ability to be easily implemented, its cost which is not very expensive and the unnecessary contact with the part to be controlled. The increasing complexity of the structures to be controlled and the augmented requests in terms of capacity of detection, particularly in terms of reachable depth and orientation of flaws as well as on the dynamics of measurement and space resolution, have shown the limits of most of the actual EC sensors.

To extend the performance of detection in terms of accessible depth, signal ratio to noise and space resolution, it is useful to have a close understanding of measurement problems involved in the EC testing systems. Indeed the simplicity of implementing NDE by $\mathrm{EC}$, because of the simplicity to create these currents by means of an inductor and to detect the induced field by a sensor should not cover up the very complex task associated to optimizing the configuration of a highly effective EC based probe. The challenge is about in fact, for a given control to be carried out, how to determine the optimal distribution of the EC probe making it possible to get the best signal indication of the defect. Many parameters enter in fact in the problem and it is suitable to carry out simulations which make enable to resolve the influence of each factor on the quality of the obtained indication.

In this work, modelling is performed for the EC generated in a homogeneous and isotropic perfect conductive mass, when subjected to the action of a stationary induction field created by a feed circuit. The modelling is conducted by means of the finite element method under Comsol software package [6]. Key parameters that include, the frequency of work, the distance separating the coil sensor form the tested sample are taken into account. Using a B-scan sweeping, the presence of a small defect having the shape of an open cavity is shown to be well indicated via monitoring the variations affecting the distribution of the EC. This opens the way to perform a parametric study in order to achieve optimization of the probe and to improve further the capacity of this system in detecting small flaws having various orientations.

\section{Modelling eddy-current field in a perfect conductive material}

Maxwell equations for a perfect conductive material are of the form

$$
\nabla . D=\rho, \nabla . B=0, \nabla \wedge E=-\frac{\partial B}{\partial t} \text { and } \nabla \wedge H=J+\frac{\partial D}{\partial t}
$$

where $B$ is the inductive magnetic field, $D$ the electric displacement, $H$ the magnetic excitation vector, $E$ the electric field, $\rho$ the density of charges and $J$ the current density.
In a perfect Ohmic homogeneous and isotropic conductive medium, the constitutive equations take the following form

$J=\sigma E, D=\varepsilon E$ and $B=\mu H$

where $\sigma$ is the electric conductivity of the medium, $\varepsilon$ the permittivity and $\mu$ the permeability.

In case of a metallic material, the distribution of charges vanishes quickly due to the high conductivity and it is quite admissible to assume the hypothesis that $\rho=0$. A decoupling can then be operated in the Maxwell system of equations (1), to obtain the following propagation-diffusion equations

$\Delta E-\varepsilon \mu \frac{\partial^{2} E}{\partial t^{2}}-\mu \sigma \frac{\partial E}{\partial t}=0, \Delta B-\varepsilon \mu \frac{\partial^{2} B}{\partial t^{2}}-\mu \sigma \frac{\partial B}{\partial t}=0$

Let's consider a stationary and harmonic regime which is occurring at the radial frequency $\omega$ for which $E=E_{0} e^{j\left(\omega t-\varphi_{E}\right)}$ and $B=B_{0} e^{j\left(\omega t-\varphi_{B}\right)}$, where $E_{0}$ and $B_{0}$ are quantities that depend only on the space variables, $t$ designates the time, $\varphi_{E}$ and $\varphi_{B}$ the phases at the origin of time and $j$ the unitary pure imaginary. In practice, one can easily verify that the following condition $\omega \omega_{c}$ where $\omega_{c}=\sigma / \varepsilon$ holds for metallic materials. In this case, equations (3) simplify to their diffusive parts as

$\Delta E_{0}-j \mu \omega \sigma E_{0}=0, \Delta B_{0}-j \mu \omega \sigma B_{0}=0$

Equations (2) and (4) yield the existence of a permanent density of currents that are permanently circulating in the conductive material and having the form $J=\sigma E_{0} e^{j\left(\omega t-\varphi_{E}\right)}$. These are called eddy-currents (EC).

For a given sample geometry, the field $J$ can be computed by integrating equations (4) under some specified boundary conditions. In the presence of a singularity or a flaw in the medium, the conductivity varies and the EC distribution will manifest changes as compared to that one associated to the intact geometry. This gives hence the possibility of using these EC to monitor flaw detection in metallic parts.

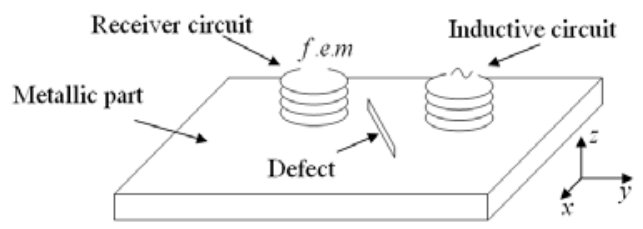

Fig. 1. Schematic representation of a probe-coil system that uses the EC principle.

The implementation of this technique of diagnosis sets usually on two circuits, figure 1. The first circuit constitutes the inductive which puts a stationary variable magnetic field in the tested material. The second circuit takes the form of a sensor which consists of an open 
circuit that is influenced by the variable magnetic field created by the EC. This can provide thus the defect indication as a perturbation endured by the electric tension while the sensor is crossing a zone with variable local conductivity.

The material is excited by a circulating current in the inductive coil and an induced voltage in the receiver coil which defines the sensor. This voltage is proportional to the intensity of EC field developing in the skin of the material. If the induction field depends only on the $z$ coordinate (counted starting from the material surface), assuming that the material can be viewed to be a semiinfinite medium, solution of equations (4) can be conducted analytically and one finds in real notations the following distribution of the EC [7]

$J(z, t)=B_{0} \sqrt{\frac{\sigma \omega}{2 \mu}} \exp \left(-z \sqrt{\frac{\mu \sigma \omega}{2}}\right) \cos \left(\omega t-z \sqrt{\frac{\mu \sigma \omega}{2}}+\frac{\pi}{4}\right)$

It could be seen thus the intensity of the EC depends on the depth of the considered point. The penetrating depth denoted $\delta$ correspond to the top layer of the material where the skin effect manifests strongly itself under a variable excitation regime. The penetrating depth correspond to the point where the intensity is that at the surface divided by the Neper number $e$ which yields then $\delta=1 / \sqrt{\pi f \mu \sigma}$ where $f=\omega /(2 \pi)$ is the work frequency. In practice, the excitation frequency can be monitored to reach deep points of the material, but also the magnitude of the inductive magnetic field $B_{0}$ can be increased by using a coil containing a high number of whorls. This shows the advantage to work with low frequencies for which the condition $\omega \omega_{c}$ is easily satisfied. Validity of equations (4) to model the EC phenomenon is then stated.

For a material whose geometry can not be assimilated to a semi-infinite medium, or presenting irregularities at its surface solution of equations (4) can be achieved by means of the finite element method. In the following Comsol software packages is used to model a testing setup having the form of system shown in figure 1, [8].

\section{Results and discussion}

Let's consider a rectangular plate made form aluminium and having the following geometric dimensions: length $L=0.8 m$, width $\ell=0.4 m$ and height $h=0.01 \mathrm{~m}$, figure 2 . The plate material has the conductivity $\sigma_{A l}=3.774 \times 10^{7} S . m^{-1}$ and the relative permeability can be taken to be $\mu_{r}=1 H . m^{-1}$.

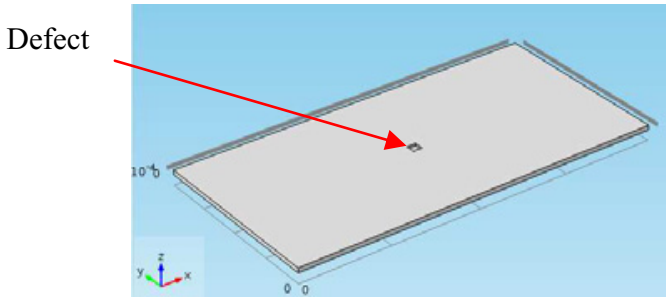

Fig. 2. Sample geometry developed under Comsol.
A defect having the form of an open parallelepiped cavity which sides are parallel to those of the plate is assumed to be present at the material surface at the location indicated in figure 2 . Its dimensions correspond to length $L_{d}=20 \mathrm{~mm}$, width $\ell_{d}=20 \mathrm{~mm}$ and depth $h_{d}=5 \mathrm{~mm}$.

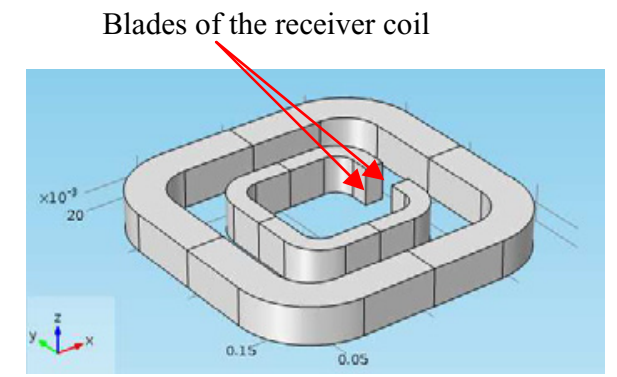

Fig. 3. Details of the inductive (external part) and the receiver (internal part) coils.

The inductive coil is made from cupper for which the electric conductivity is $\sigma_{C u}=6 \times 10^{7} \mathrm{~S} . \mathrm{m}^{-1}$ and the relative permeability can gain be taken to be $\mu_{r}=1 H \cdot m^{-1}$, the external part in figure 3 . The coil base is square with span $0.16 \mathrm{~m}$. This coil is assumed to be formed by 1000 whorls of transverse section $a_{c}=10^{-6} \mathrm{~m}^{2}$. It is assumed to be crossed by a circulating sinusoidal current having the maximum intensity $I_{c}=1 \mathrm{~A}$. Two work frequencies are selected $f_{1}=50 \mathrm{~Hz}$ and $f_{2}=100 \mathrm{~Hz}$.

The sensor coil is placed inside the inductive coil and is made also from cupper. This takes the form of an open circuit enabling to measure the voltage between the blades, the internal part in figure 3 . The coil base is square and has a span of $0.08 \mathrm{~m}$.

Both the inductive and the sensor coils are placed at a distance $\eta=20 \mathrm{~mm}$ from the top surface of the plate assumed to be perfectly smooth and planar.

A finite element model was derived under the code Comsol Multiphysics [7]. Figure 4 gives the considered mesh corresponding to the finite element convergence. The mesh was generated automatically by using free tetrahedral elements; its maximum size was $0.02 \mathrm{~m}$ for the plate and $0.01 \mathrm{~m}$ for the coils. The tree elements constituting the probe-coil set-up are meshed using the same element type. As the excitation coil radiates electromagnetic waves through air according to equations (3) before these can reach the plate material, the environmental air should also be modelled.

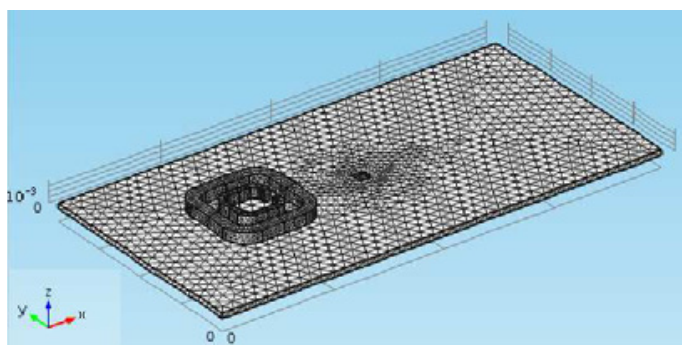

Fig. 4. Mesh generated for the plate and the coils. 
Because meshing an infinite volume is not possible, it is necessary to specify a finite volume to mesh and solve for it. In the present case, the whole probe-coil setup was placed at the centre of a cube as shown in figure 5(a). To avoid reflection that is susceptible to take place in a finite volume, the default boundary condition Magnetic Insulation is used. This condition forces the field to be tangential to the exterior boundaries. The cube used here is sufficiently large its edge is chosen to have a length equal to $2 \mathrm{~m}$. Figure 5 (b) shows the mesh developed under Comsol for this cube. To complete the material properties, the air electric conductivity is fixed at $\sigma_{\text {air }}=10 S . \mathrm{m}^{-1}$.

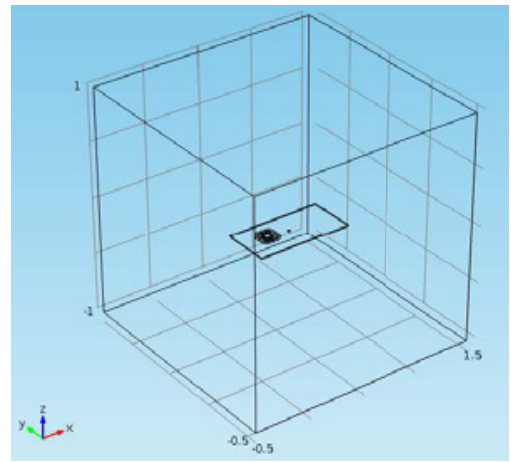

(a)

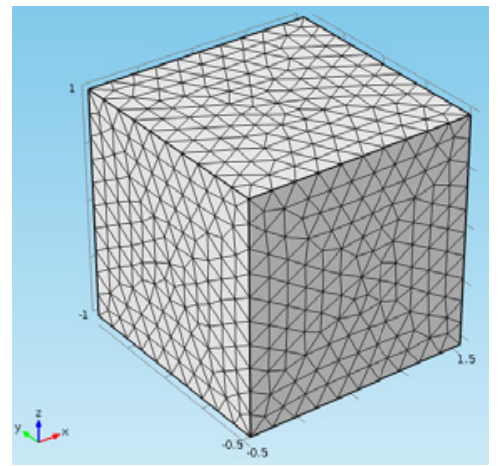

(b)

Fig. 5. The complete model of the EC probe set-up;

(a) Location of the plate and the coils inside the cube; (b) Cube mesh.

Considering a cut-line that crosses the defect at its centre (as shown in red colour in figure 6), four positions of the coils were considered along this line to enable performing a basic B-scan. The idea is that sensitivity of results to the defect will vary according to the distance separating the coils from the defect location. Figure 6 presents the selected sequence of these positions. The first position corresponds to the centre placed at the distance $0.22 \mathrm{~m}$ from the defect position. The subsequent positions correspond to successive incremental translations of $0.1 \mathrm{~m}$ magnitude.

The intensity of the EC as generated by the excitation coil while working consecutively with the frequencies $f_{1}=50 \mathrm{~Hz}$ and $f_{2}=100 \mathrm{~Hz}$ was calculated by means of the finite element model. Internal boundary condition corresponding to electric and magnetic surfaces where applied, and the density of charge was fixed at zero.

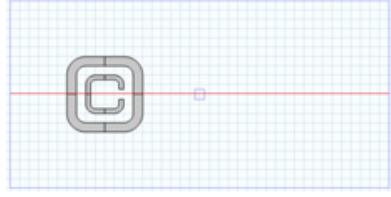

(a)

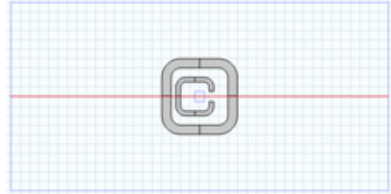

(c)

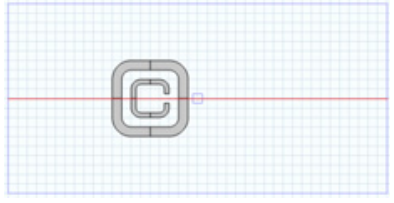

(b)

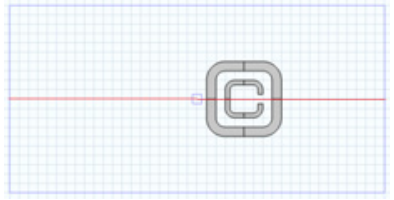

(d)
Fig. 6. The four positions of the coils considered in simulations.

Figure 7 shows contours of the EC associated to the $y$-component of EC density vector and located at the surface of the defected plate.

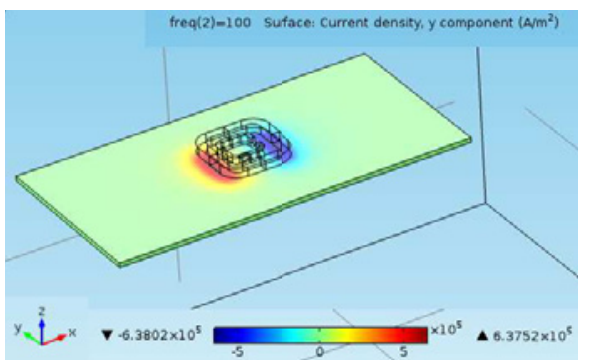

Fig. 7. Contours of the EC density vector component along the $y$ axis; the coils are placed right on the defect.

Figure 8 presents the obtained cut-line of induced current density for the four positions of the coils as given in figure 6. One can see that a sudden decrease of intensity appears at the defect zone. This discontinuity indicates the presence of a defect. One can notice however that, when the coils are too far or centred right on the defect, the perturbation associated to the defect is small and cannot be easily detected when using for instance current probes prospecting the plate surface along the cut-line. The ideal positions to obtain maximum effect corresponds in the present case to positions between positions 2 and 3 or positions 3 and 4 .

Figure 8 shows also that increasing frequency will increase the EC magnitude. However the difference between the two frequencies vanishes when crossing the defect zone as the two work frequencies give almost the same result. One can conclude then that using $f_{1}=50 \mathrm{~Hz}$ is quite sufficient. Moreover depth of penetration associated to this frequency will be higher.

Scanning the plate along a cut line with probes is not the best practical solution because of contact problems that will arise and the irregularities present on the plate surface which will perturb measurement. Moreover a single cut-line would not be sufficient and a C-scan strategy would be required. This is way a receiver coil is generally used to get more easily the effect resulting from the presence of a flaw defect in the inspected plate.

The receiver coil shown in figure 3 is an open circuit and the blades will show an electric volage as a result of the magnetic field created by the EC in the plate. This 
voltage varies with the position of the point on the blade. The corner points of these blades were chosen as measurement points. Figure 9 shows the labelling used for these points. Connecting these points with a voltage measuring instrument can be used to monitor the information about the existence of a defect. This can be performed in comparison with a defect free state of the plate. The obtained voltages in the eight points for both defect free and defected plate were processed to give the maximum variation of voltage when the coils are displaced according to the three transitions: (a) $\rightarrow$ (b); (b) $\rightarrow$ (c) and (c) $\rightarrow$ (d). The obtained results are given in figure 10. The maximum values for each transition are summarized in table 1.

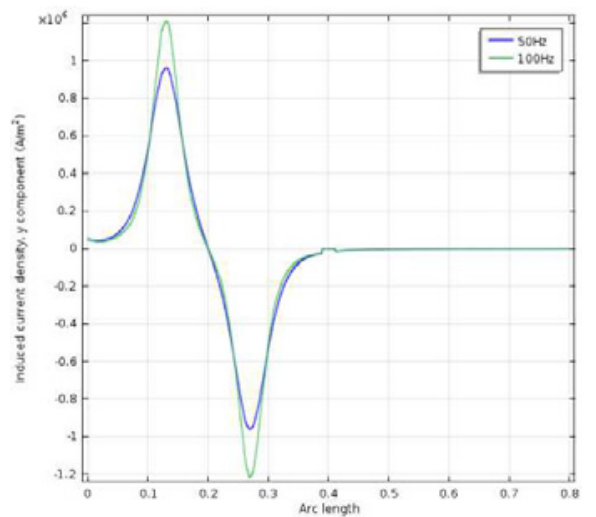

(a)

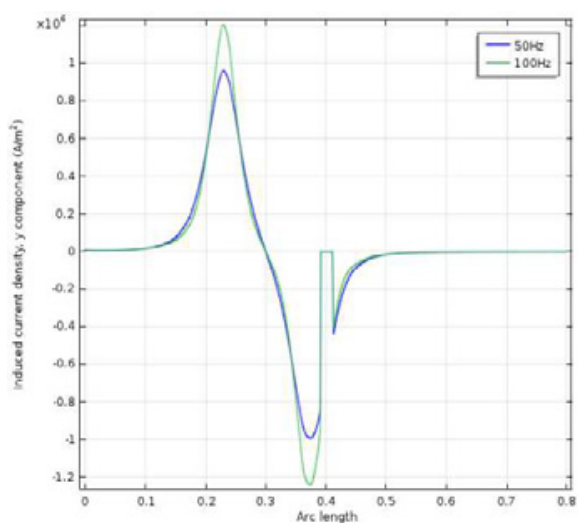

(b)

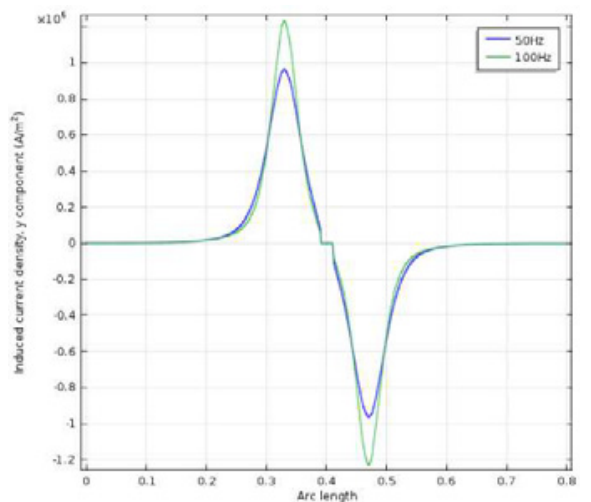

(c)

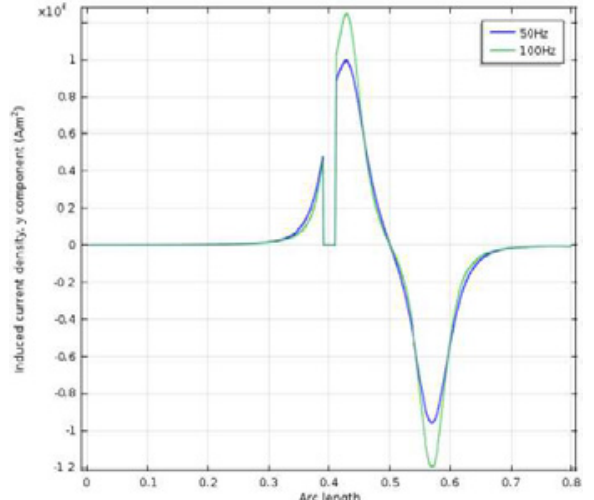

(d)

Fig. 8. Cut lines giving the $y$ component of induced current density for the four positions of the coils and the two work frequencies $f_{1}=50 \mathrm{~Hz}$ (in blue) and $f_{2}=100 \mathrm{~Hz}$ (in green).

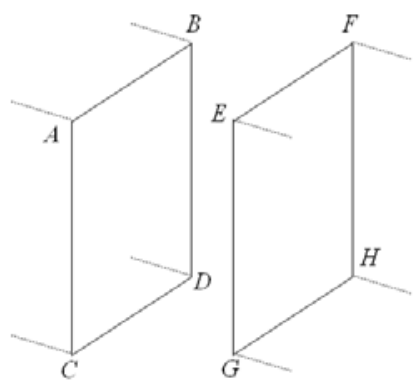

Fig. 9. Designation of point corners on the blades of the receiver coil.

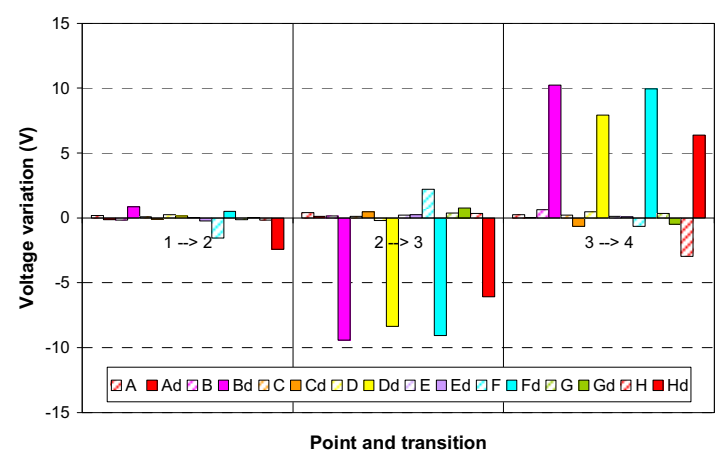

Fig. 10. Voltage variations as function of the point on the blade and the transition between coil positions; red colour corresponds to the configuration of the plate with a defect..

Table 1. Maximum voltage variation as function of the transition between two successive positions of the coils on the cut-line.

\begin{tabular}{|c|c|c|}
\hline $\begin{array}{c}\text { Coils } \\
\text { transition }\end{array}$ & $\begin{array}{c}\text { Maximum } \\
\text { voltage variation } \\
\text { without defect }\end{array}$ & $\begin{array}{c}\text { Maximum } \\
\text { voltage variation } \\
\text { with defect }\end{array}$ \\
\hline (a) $\rightarrow$ (b) & 0.169 & 0.87 \\
\hline (b) $\rightarrow$ (c) & 0.163 & 9.419 \\
\hline (c) $\rightarrow$ (d) & 0.651 & 10.231 \\
\hline
\end{tabular}


From figure 10, one can see that by monitoring voltage variations on the surface of the blades, it is quite possible to get information about the presence of a defect. From table 1, one can observe that the highest variations associated to the plate configuration with a defect are at least from 5 to 58 times greater than those of a plate without defect. These variations admit peaks when the coils are in the vicinity of the zone where the defect is located.

Optimisation of the system and reliability of results has still to be performed. One of the important problems that could limit reliability of the system is its sensitivity to the distance separating the coils from the plate which can vary because of roughness of the plate surface.

\section{Conclusions}

Non destructive evaluation based on eddy currents has been investigated in this work in the particular case of surface like defect located on a plate. Finite element modelling of the problem was performed under Comsol software package where the electromagnetic stationary solution was calculated. Post-processing of the obtained results has shown that detection of defects can be performed by rather using the information collected on voltage variation as experienced in different points of the receiver coil. The present finite element model can be used to further investigate detectability of small defects and to assess reliability of detection as it could be affected by uncertainties due to the part profile and voltage measurement on the receiver coil.

\section{References}

1. C.E. Betz, Principles of Magnetic Particle Testing (American Society for Nondestructive Testing, 1985)

2. L. Rosado, T.G. Santos, P.M. Ramos, P. Vilac., M. Piedade, NDT\&E International 51 (2012)

3. P. Xu, S. Huang, W. Zhao, NDT\&E International 44 (2011)

4. M. Ur Rahman, Optimization of in-line defect detection by eddy current techniques ( $\mathrm{PhD}$ Thesis, Kassel University, Germany, 2010)

5. A. Rosell, G. Persson, International Journal of Fatigue 41 (2012)

6. Anonymous, Introduction to Comsol Multiphysics, Part number: CM010004, Version: November (Comsol, 2013)

7. C.V. Dodd, W.E. Deeds, Journal of Applied Physics, 39(6) (1968)

8. G.L. Hower, L.D. Philipp, A. Abathi, R.W. Rupe, Journal of Nondestructive Evaluation, 4(1) (1984) 